\title{
A Mathematical Model for the Response of Immune Cells to Mycobacterium Tuberculosis
}

\author{
Fitri Nur Rahmawati, Yudi Ari Adi* \\ Department of Mathematics, Faculty of Applied Science and Technology, Ahmad Dahlan University, Yogyakarta, Indonesia.
}

How to cite this paper: Fitri Nur Rahmawati, Yudi Ari Adi. (2021) A Mathematical Model for the Response of Immune Cells to Mycobacterium Tuberculosis. Journal of Applied Mathematics and Computation, 5(1), 1-8.

DOI: 10.26855/jamc.2021.03.001

Received: November 30, 2020

Accepted: December 27, 2020

Published: January 20, 2021

*Corresponding author: Yudi Ari Adi, Department of Mathematics, Faculty of Applied Science and Technology, Ahmad Dahlan University, Yogyakarta, Indonesia.

Email: yudi.adi@math.uad.ac.id

\begin{abstract}
Tuberculosis (TB) is an infectious disease that is a problem almost all over the world. In 2019, the World Health Organization (WHO) reported 10 million new infections each year, with an average of 1.2 million people dying from the disease. Vaccination to healthy people is an effort to protect against infection with this disease. In this paper, a mathematical model of the interaction of the immune response against Mycobacterium tuberculosis with vaccine administration is studied. The model is in the form of a system of ordinary differential equations with four variables. Furthermore, an analysis of the stability of the equilibrium point is carried out. The results obtained indicate that the disease-free equilibrium point is globally asymptotically stable if $R_{0} \leq 1$, and unstable if $R_{0}>1$. The sensitivity analysis showed that the infection rate and the bacterial growth rate were the two most influencing factors for the infection's survival. This study's results are expected to be reference doctors and paramedics to reduce tuberculosis cases.
\end{abstract}

\section{Keywords}

Mycobacterium tuberculosis, macrophage, a mathematical model

\section{Introduction}

Tuberculosis (TB) is an infectious disease caused by the bacteria Mycobacterium tuberculosis, which can attack the lungs or other body organs. Tuberculosis is one of the top 10 causes of death worldwide. WHO (2019) reported that India is the largest contributor to TB cases in the world, amounting to $27 \%$. Five other countries that account for nearly two-thirds of total global TB cases are China (9\%), Indonesia (8\%), the Philippines (6\%), Pakistan (6\%), Nigeria (4\%) [1].

TB is characterized by early fever symptoms, a relatively long cough, coughing up blood, and chest pain [2]. This disease is transmitted through the air containing the bacteria Mycobacterium tuberculosis, not through direct contact. When people with active TB experience coughing, sneezing, screaming, or singing, the bacteria will be carried out of the lungs into the air. Transmission occurs when a person inhales air containing the bacteria Mycobacterium tuberculosis and then reaches the lungs' alveoli. When bacteria enter the human body, the immune system will try to resist and destroy them. The immune response plays a role in controlling the development of Mycobacterium tuberculosis in macrophages and $\mathrm{T}$ lymphocytes [3].

Macrophages originate from monocytes, which are part of white blood cells. These macrophages include phagocytic cells (eating cells). Cells damaged by bacteria will produce chemical signals that function to call phagocytes (macrophages) to eat or destroy these bacteria. T lymphocytes are white blood cells that are part of the immune system. The role of T lymphocytes is to recognize harmful foreign objects that enter the body or antigens. With memory T lymphocytes, antigens that have entered the body can be easily identified and destroyed more quickly [4].

Mathematical models can be applied to understand tuberculosis dynamics. Several mathematical models have been 
developed to study tuberculosis's dynamics, as in [5-10]. Ibarguen-Mondragon et al. [5] have modeled immunological cells in tuberculosis. Furthermore, Ibarguen et al. [6] extended the assumption of bacterial population growth by forming a system of ordinary differential equations, a model of the interaction between uninfected macrophages, infected macrophages, $\mathrm{T}$ cells, and Mycobacterium tuberculosis bacteria to control tuberculosis. We performed numerical simulations by entering values in the model parameters to estimate the likelihood of disease. Shi et al. [7] extended the previous model to consider chemotherapy drug administration. They were using assumptions that the rate of chemotherapy drug administration is constant (fixed), and the rate of chemotherapy drug administration varies.

Meanwhile, Magombedze et al. [8] developed a tuberculosis model with the infection's location in the lungs. Zhang et al. [9] established a tuberculosis model and then performed global stability and bifurcation analysis for disease-free equilibrium points. Also, Adi and Thobirin [10] compiled a macrophage cell and Tb bacteria interactions model and found a backward bifurcation condition. This paper constructs a dynamic model of tuberculosis in a within-host, an extension of the Adi and Thobirin model in [10], taking vaccination into account.

We organize the paper as follows. In Section 2, we formulate our model and establishing the properties of solutions. We then discuss the existence of equilibria and the stability of the disease-free equilibrium point in Section 3 . In section 4 , we provide some numerical simulations to support the theoretical results. Finally, the discussion and conclusion are given in Section 5.

\section{Mathematical model}

To construct a mathematical model of the immune response of tuberculosis bacteria, we divide the population into uninfected macrophages $\left(M_{u}\right)$, infected macrophages $\left(M_{i}\right)$, Mycobacterium tuberculosis (B) and T cells (T). It is assumed that uninfected macrophages reproduce with a growth or recruitment rate of uninfected macrophages of $\Lambda_{u}$ and die with a natural mortality rate of uninfected macrophages of $\mu_{u}$. When bacteria enter the body, uninfected macrophages will get a signal to come to cells that are attacked by bacteria. There is a possibility that uninfected macrophages are unable to clean the bacteria so that uninfected macrophages will become infected macrophages with an infected rate of $\beta$. The infected macrophages were killed by T cells of $\alpha_{T}$ and the infected macrophages exploded due to the proliferation of bacteria outside the threshold for infected macrophages of $b$.

Mycobacterium tuberculosis bacteria multiply in infected macrophages to a threshold and then explode and release the bacteria. Therefore, it is assumed that the growth rate of $r b M_{i}$ with $r$ is the rate of bacteria reproducing in infected macrophages. The released bacteria will become extracellular, then infect macrophages or be eaten (killed) by uninfected macrophages $\gamma$ and the natural death rate of bacteria is $\mu_{B}$. The $\mathrm{T}$ cell population follows a logistic equation $(1-T) k_{1} M_{i}$, where $k_{1}$ is the growth rate of T cells and can be stimulated by administration of vaccination, $\frac{c_{B}}{e_{B} T+1}$ and died with a natural death rate $\mu_{T}$.

From the above assumptions, the TB model is written as follows

$$
\begin{aligned}
& \frac{d M_{u}}{d t}=\Lambda_{u}-\beta B M_{u}-\mu_{u} M_{u} \\
& \frac{d M_{i}}{d t}=\beta B M_{u}-b M_{i}-\alpha_{T} M_{i} T \\
& \frac{d B}{d t}=r b M_{i}-\gamma M_{u} B-\mu_{B} B \\
& \frac{d T}{d t}=(1-T) k_{1} M_{i}+\frac{c_{B} B T}{e_{B} T+1}-\mu_{T} T
\end{aligned}
$$

The following theorem verifies that all solutions with nonnegative initial conditions remain nonnegative for all $t>0$. So the model of the system (1) has biological meaning.

Theorem 1. The set

$$
\Omega=\left\{\left(M_{u}, M_{i}, B, T\right) \in \mathbb{R}_{+}^{4} \mid M_{u}+M_{i} \leq \frac{\Lambda_{u}}{\mu_{u}}, B \leq \frac{r b \Lambda_{u}}{\mu_{u}}, T \leq \frac{\Lambda_{u}}{\mu_{T} \mu_{u}}\left(k_{1}+\frac{r b c_{B}}{e_{B}}\right)\right\},
$$

is a positive invariant and attracting set for equation (1).

Proof. From the first and second equation of (1), we find

$$
\frac{d M_{u}}{d t}+\frac{d M_{i}}{d t}=\Lambda_{u}-\mu_{u} \mathrm{M}_{u}-b \mathrm{M}_{i} \leq \Lambda_{u}-\bar{\mu}_{\mathrm{u}}\left(\mathrm{M}_{u}+\mathrm{M}_{i}\right)
$$

with $\bar{\mu}_{\mathrm{u}}=\min \left\{\mu_{u}, b\right\}$. We have 
From the third equation of (1), we find

$$
0 \leq\left(\mathrm{M}_{u}+\mathrm{M}_{i}\right)(t) \leq \frac{\Lambda_{u}}{\bar{\mu}_{\mathrm{u}}}=M_{\max } .
$$

Then, we have

$$
\frac{d B}{d t}=r b M_{i}-\gamma M_{u} B-\mu_{B} B \leq r b \frac{\Lambda_{u}}{\bar{\mu}_{\mathrm{u}}}-\mu_{B} B
$$

Lastly, from the fourth equation of (1), we have

$$
B(t) \leq \frac{r b M_{\max }}{\mu_{B}}=B_{\max } .
$$

$$
\frac{d T}{d t}=(1-T) k_{1} M_{i}+\frac{c_{B} B T}{e_{B} T+1}-\mu_{T} T \leq(1-T) k_{1} \frac{\Lambda_{u}}{\bar{\mu}_{\mathrm{u}}}+\frac{c_{B}}{e_{B}} \frac{r b M_{\max }}{\mu_{B}}-\mu_{T} T .
$$

Hence,

$$
\lim \sup _{t \rightarrow \infty} T(t) \leq \frac{1}{\mu_{T}}\left(k_{1} M_{\max }+\frac{c_{B}}{\varepsilon_{B}} B_{\max }\right) .
$$

Therefore, all solutions of model (1) that initially in $\Omega$ remain in $\Omega$ for all $t \geq 0$.

\section{Existence and stability of equilibrium points}

The system (1) always have a disease-free equilibrium point $E_{0}=\left(\frac{\Lambda_{u}}{\mu_{u}}, 0,0,0\right)$. Then, applying the next generation matrix procedures, we calculate the basic reproduction number as follows. The rate of new infections $\mathscr{F}$ and the rate of transfer in and out of the two compartments $\mathcal{V}$ are

$$
\mathscr{F}=\left[\begin{array}{c}
\beta B M_{u} \\
0
\end{array}\right], \quad \mathcal{V}=\left[\begin{array}{c}
b M_{i}+\alpha_{T} M_{i} T \\
-r b M_{i}+\gamma M_{u} B+\mu_{B} B
\end{array}\right] .
$$

The Jacobian matrices of $\mathscr{F}$ and $\mathcal{V}$ at the disease-free equilibrium are

$$
F=\left[\begin{array}{cc}
0 & \frac{\beta \Lambda_{u}}{\mu_{u}} \\
0 & 0
\end{array}\right] \quad V=\left[\begin{array}{cc}
b & 0 \\
-r b & \frac{\gamma \Lambda_{u}}{\mu_{u}}+\mu_{B}
\end{array}\right] .
$$

Therefore, the basic reproduction number, $R_{0}$ is the spectral radius of the matrix $F V^{-1}$, and hence $R_{0}$ is

Furthermore, we have the following theorem.

$$
R_{0}=\frac{r \beta \Lambda_{u}}{\gamma \Lambda_{u}+\mu_{u} \mu_{B}} .
$$

Theorema 2. If $R_{0}<1$ then the system (1) has an only disease-free equilibrium point $E_{0}=\left(\frac{\Lambda_{u}}{\mu_{u}}, 0,0,0\right)$ and if $R_{0}>1$ then the system (1) has two equilibrium points, that is, the disease-free equilibrium point $E_{0}=\left(\frac{\Lambda_{u}}{\mu_{u}}, 0,0,0\right)$ and the disease equilibrium point $E_{1}=\left(M_{u}^{*}, M_{i}^{*}, B^{*}, T^{*}\right)$.

Proof. Solving the system (1) by setting the right-hand side equal to zero, we get

$$
M_{u}^{*}=\frac{\Lambda_{u}}{\beta B+\mu_{u}}, M_{i}^{*}=\frac{B}{r b}\left[\gamma \frac{\Lambda_{u}}{\beta B+\mu_{u}}+\mu_{B}\right], T_{1}^{*}=\frac{k_{1} B}{r b}\left[\gamma \frac{\Lambda_{u}}{\beta B+\mu_{u}}+\mu_{B}\right],
$$

and $B^{*}$ is a positive real root of

where

$$
a_{1} B^{4}+a_{2} B^{3}+a_{3} B^{2}+a_{4} B=0,
$$

$$
\begin{gathered}
a_{1}=\frac{\beta^{2} k_{1}\left(\mu_{B}\right)^{2} \alpha_{T}}{(r b)^{2}} \\
a_{2}=2 \frac{\beta k_{1} \gamma \Lambda_{u} \alpha_{T} \mu_{B}}{(r b)^{2}}+\frac{\beta^{2} \mu_{B}}{r}+\frac{\beta k_{1} \mu_{u}\left(\mu_{B}\right)^{2} \alpha_{T}}{(r b)^{2}}+\frac{\beta \mu_{u} k_{1}\left(\mu_{B}\right)^{2} \alpha_{T}}{(r b)^{2}} \\
a_{3}=\frac{r b^{2} \beta}{(r b)^{2}}\left(\gamma \Lambda_{u}+\mu_{u} \mu_{B}\right)\left(1-R_{0}\right)+\frac{k_{1}\left(\gamma \Lambda_{u}\right)^{2} \alpha_{T}}{(r b)^{2}}+2 \frac{k_{1} \gamma \Lambda_{u} \alpha_{T} \mu_{u} \mu_{B}}{(r b)^{2}}+\frac{\beta \mu_{u} \mu_{B}}{r}+\frac{k_{1}\left(\mu_{u} \mu_{B}\right)^{2} \alpha_{T}}{(r b)^{2}} \\
a_{4}=\frac{\mu_{u}}{r}\left(\gamma \Lambda_{u}+\mu_{u} \mu_{B}\right)\left(1-R_{0}\right) .
\end{gathered}
$$

We find $B=0$ or 


$$
a_{1} B^{3}+a_{2} B^{2}+a_{3} B+a_{4}=0 .
$$

If $B=0$ we have a disease-free equilibrium point, $E_{0}=\left(\frac{\Lambda_{u}}{\mu_{u}}, 0,0,0\right)$.

The coefficient $a_{1}$ and $a_{2}$ always positive. If $R_{0}<1$, the coefficient $a_{3}$ and $a_{4}$ also positive, so there no positive real root. Consequently, system (1) has no disease equilibrium point. If $R_{0}>1$, then the coefficient $a_{3}$ can be positive or negative, while $a_{4}$ is negative. Hence, there is one change of coefficient sign of (2), and acco rding to Descartes's rule of sign, there is at least one positive root of (2). We find that, in addition to $E_{0}$, the system (1) at least has one positive disease equilibrium point. This completes the proof.

Next, the following theorem shows the equilibrium point's stability determined by the sign of real part eigenvalues of the Jacobian matrix of system (1) at the equilibrium point.

Theorema 3. The disease-free equilibrium $E_{0}$ is locally asymptotically stable if $R_{0}<1$ and unstable if $R_{0}>1$.

Proof. The Jacobian matrix of system (1) at $E_{0}=\left(\frac{\Lambda_{u}}{\mu_{u}}, 0,0,0\right)$ is

The characteristic polynomial of $J_{0}$ is

$$
J_{0}=\left[\begin{array}{cccc}
-\mu_{u} & 0 & -\frac{\beta \Lambda_{u}}{\mu_{u}} & 0 \\
0 & -b & \frac{\beta \Lambda_{u}}{\mu_{u}} & 0 \\
0 & r b & -\frac{\gamma \wedge_{u}}{\mu_{u}}-\mu_{B} & 0 \\
0 & k_{1} & 0 & -\mu_{T}
\end{array}\right] .
$$

$$
p(\lambda)=\left(\lambda+\mu_{u}\right)\left(\lambda+\mu_{T}\right)\left(\lambda^{2}+\left(b+\mu_{B}+\frac{\gamma \wedge_{u}}{\mu_{u}}\right) \lambda+\left(\frac{b \gamma \wedge_{u}}{\mu_{u}}+b \mu_{B}\right)\left(1-R_{0}\right)\right)=0 .
$$

The roots of (3) are $\lambda_{1}=-\mu_{u}<0, \lambda_{2}=-b<0$, and the other two roots are determined by the quadratic equation

$$
\lambda^{2}+\left(b+\mu_{B}+\frac{\gamma \wedge_{u}}{\mu_{u}}\right) \lambda+\left(\frac{b \gamma \wedge_{u}}{\mu_{u}}+b \mu_{B}\right)\left(1-R_{0}\right)=0
$$

We find that both roots of equation (4) have a negative real part if $R_{0}<1$. If $R_{0}>1$, the equation (4) has one root with a positive real part. Hence, $E_{0}$ is locally asymptotically stable if $R_{0}<1$ and unstable if $R_{0}>1$.

We then prove the global stability of $E_{0}$ when $R_{0} \leq 1$.

Theorema 4. The disease-free equilibrium $E_{0}$ is globally asymptotically stable if $R_{0} \leq 1$.

Proof. Consider the Lyapunov function

$$
V=\beta \Lambda_{u} B+\left(\gamma \Lambda_{u}+\mu_{u} \mu_{B}\right) M_{i}
$$

The derivative $V$ given by

$$
\begin{gathered}
\frac{d V}{d t}=\beta \Lambda_{u}\left(r b M_{i}-\gamma M_{u} B-\mu_{B} B\right)+\left(\gamma \Lambda_{u}+\mu_{u} \mu_{B}\right)\left(\beta B M_{u}-b M_{i}-\alpha_{T} M_{i} T\right) \\
=r \beta \Lambda_{u} b M_{i}-\gamma \beta \Lambda_{u} M_{u} B-\beta \Lambda_{u} \mu_{B} B+\gamma \Lambda_{u} \beta B M_{u}-b \gamma \Lambda_{u} M_{i}-\gamma \Lambda_{u} \alpha_{T} M_{i} T \\
\quad+\mu_{u} \mu_{B} \beta B M_{u}-\mu_{u} \mu_{B} b M_{i}-\mu_{u} \mu_{B} \alpha_{T} M_{i} T \\
\leq\left(\gamma \Lambda_{u}+\mu_{u} \mu_{B}\right) b M_{i}\left(R_{0}-1\right)+\mu_{B} \beta \mu_{u} B\left(M_{u}-\frac{\Lambda_{u}}{\mu_{u}}\right) .
\end{gathered}
$$

Since $M_{u} \leq \frac{\Lambda_{\mathrm{u}}}{\mu_{u}}$, we have $\frac{d V}{d t} \leq 0$ as long as $R_{0} \leq 1$. Furthermore, we can see from the system (1) that as $t$ tend to infinity, $M_{u} \rightarrow \frac{\Lambda_{u}}{\mu_{u}}, M_{i} \rightarrow 0, B \rightarrow 0$, and $T \rightarrow 0$. Hence, the maximum invariant set in $\left\{M_{u}, M_{i}, B, T \in \Omega: \frac{d V}{d t}=0\right\}$, is the singleton $E_{0}=\left(\frac{\Lambda_{u}}{\mu_{u}}, 0,0,0\right)$. Thus, by following Lasalle's Invariance Principle in [11], we have that $E_{0}$ is globally asymptotically stable.

For the stability of disease equilibrium $E_{1}$, we claim the following theorem.

Theorema 5. Let $A=\beta B^{*}+\mu_{u} ; J=\beta B^{*} ; C=\gamma B^{*} ; D=b+\alpha_{T} T^{*} ; E=r b ; F=\beta M_{u}^{*} ; G=\gamma M_{u}^{*}+\mu_{B}$; $H=\frac{e_{B} c_{B} T^{* 2}+c_{B} T^{*}}{\left(e_{B} T^{*}+1\right)^{2}} ; I=\alpha_{T} M_{i}^{*}$. The disease equilibrium point $E_{1}$ is locally asymptotically stable if $k_{1} M_{i}^{*}-\frac{c_{B} B^{*}}{\left(e_{B} T^{*}+1\right)^{2}}-$ $k_{1} I T^{*}-k_{1} I>0, D G+A D+A G-C F-E F>0$, and $A D G+F J E-C D F-A E F>0$. 
Proof. The characteristic equation for the Jacobian matrix of system (1) at $E_{1}$ is

$$
\lambda^{4}+P_{1} \lambda^{3}+P_{2} \lambda^{2}+P_{3} \lambda+P_{4}
$$

where

$$
\begin{aligned}
& P_{1}=k_{1} M_{i}^{*}-\frac{c_{B} B^{*}}{\left(e_{B} T^{*}+1\right)^{2}}+\mu_{T}+G+D+A \\
& P_{2}=k_{1} M_{i}^{*}(+D+A)-\frac{c_{B} B^{*}}{\left(e_{B} T^{*}+1\right)^{2}}(G+D+A)+\mu_{T}(G+D+A)+(D G+A D+A G)-\left(k_{1} I T^{*}-k_{1} I\right) \\
&-(C F-E F) \\
& P_{3}= k_{1} M_{i}^{*}(D G+A D+A G)-\frac{c_{B} B^{*}}{\left(e_{B} T^{*}+1\right)^{2}}(D G+A D+A G)+\mu_{T}(D G+A D+A G)+A D G \\
&-\left(k_{1} A I T^{*}-k_{1} A I+k_{1} G I T^{*}-k_{1} G I\right)-\left(C F k_{1} M_{i}^{*}-C F \frac{c_{B} B^{*}}{\left(e_{B} T^{*}+1\right)^{2}}+C F \mu_{T}+C D F\right)+E H I+F J E \\
&-\left(E F k_{1} M_{i}^{*}-E F \frac{c_{B} B^{*}}{\left(e_{B} T^{*}+1\right)^{2}}+E F \mu_{T}+A E F\right) \\
& P_{4}=(\left.A D G k_{1} M_{i}^{*}-A D G \frac{c_{B} B^{*}}{\left(e_{B} T^{*}+1\right)^{2}}+A D G \mu_{T}\right)+\left(-k_{1} F I C+k_{1} F I C T^{*}\right)-\left(-k_{1} A G I+k_{1} A G I T^{*}\right) \\
&-\left(C D F k_{1} M_{i}^{*}-C D F \frac{c_{B} B^{*}}{\left(e_{B} T^{*}+1\right)^{2}}+C D F \mu_{T}\right)+A E H I+\left(F J E k_{1} M_{i}^{*}-F J E \frac{c_{B} B^{*}}{\left(e_{B} T^{*}+1\right)^{2}}+F J E \mu_{T}\right) \\
&-\left(A E F k_{1} M_{i}^{*}-A E F \frac{c_{B} B^{*}}{\left(e_{B} T^{*}+1\right)^{2}}+A E F \mu_{T}\right) .
\end{aligned}
$$

According to Routh-Hurwitz stability criteria, all roots of (5) have a negative real part if $P_{1}>0, P_{2}>0, P_{3}>0, P_{4}>$ 0 and $P_{1} P_{2} P_{3}>P_{3}^{2}+P_{1}^{2} P_{4}$, that is $k_{1} M_{i}^{*}-\frac{c_{B} B^{*}}{\left(e_{B} T^{*}+1\right)^{2}}-k_{1} I T^{*}-k_{1} I>0, D G+A D+A G-C F-E F>0$, and $A D G+F J E-C D F-A E F>0$.

\section{Numerical results}

In this section, we present the numerical results of the system (1). We also provide the sensitivity of the basic reproduction number $R_{0}$ to know the relative importance parameter in the disease.

\subsection{Numerical simulation}

The parameter values used in the numerical simulation were obtained from the literature [9] given in Table 1.

Table 1. Parameter values being used

\begin{tabular}{ccc}
\hline Parameter & Values & Unit \\
\hline$\Lambda_{u}$ & $1000-5000$ & ml.day $^{-1}$ \\
$\beta$ & $8,25 \times 10^{-9}-2 \times 10^{-5}$ & day $^{-1}$ \\
$\mu_{u}$ & $0,0033-0,01$ & day $^{-1}$ \\
$b$ & $0,11-0,4$ & day $^{-1}$ \\
$\alpha_{T}$ & $0,1-0,5$ & day $^{-1}$ \\
$r$ & $0,055-0,1$ & day $^{-1}$ \\
$\gamma$ & $1,25 \times 10^{-7}\left(10^{-8}, 10^{-9}\right)$ & ml. day $^{-1}$ \\
$\mu_{B}$ & 0,012 & day $^{-1}$ \\
$k_{1}$ & 0,484848 & day $^{-1}$ \\
$c_{B}$ & $5 \times 10^{-3}$ & day $^{-1}$ \\
$e_{B}$ & $10^{-4}$ & day $^{-1}$ \\
$\mu_{T}$ & $0,01-0,3333$ & day $^{-1}$ \\
\hline
\end{tabular}

We take the set of parameter values $\Lambda_{u}=1000, \beta=2 \times 10^{-6}, \mu_{u}=0.01, b=0.11, \alpha_{T}=0.1, r=0.1, \gamma=1.25 \times$ 
$10^{-7}, \mu_{B}=0.012, \mu_{T}=0.1$. With these values, we have $R_{0}=0.8163<1$. Figure 1 shows that with a different initial condition, the solution converges to the disease-free equilibrium point $E_{0}=(100000,0,0,0)$. On the other hand, by choosing $\beta=2 \times 10^{-5}$ and keeping all others parameter values, we have $R_{0}=8.163>1$, and we show the stability of the disease equilibrium point $E_{1}=(13065,4123,3327,1)$ (Figure 2).
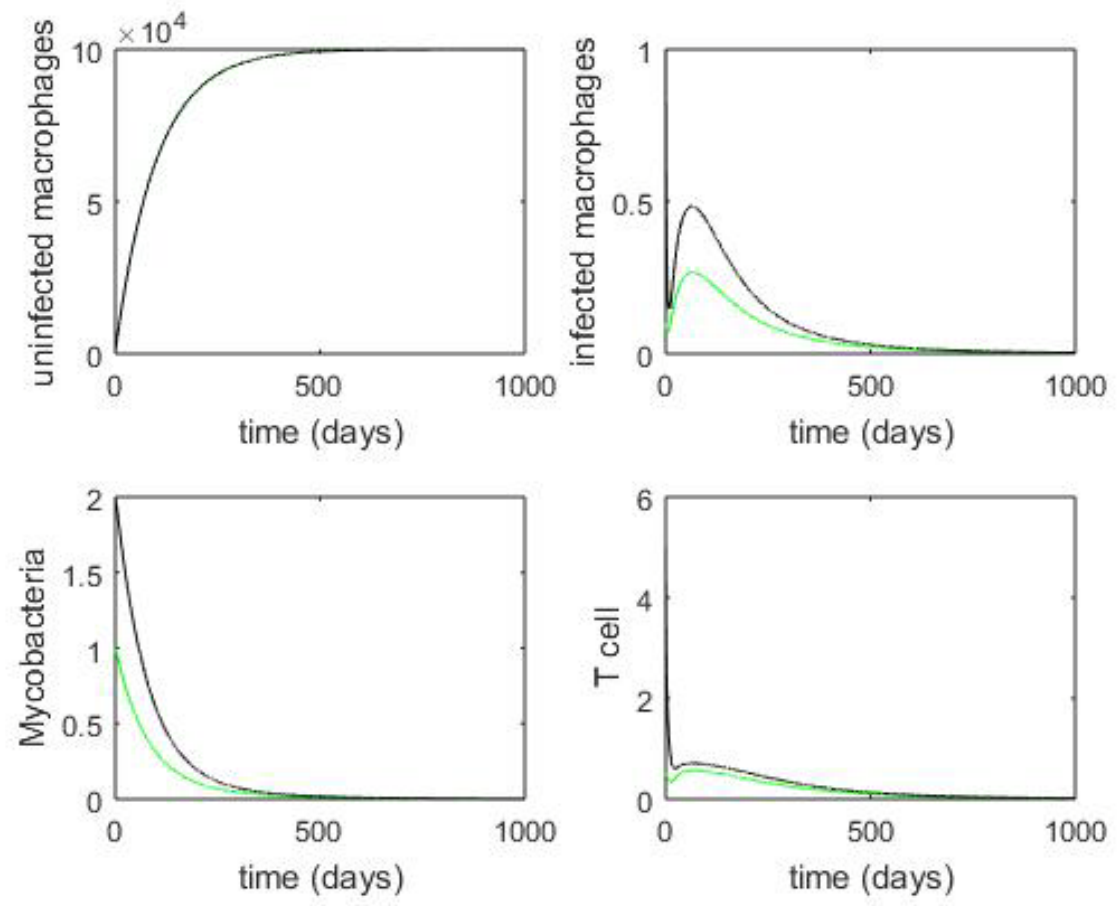

Figure 1. Time series solution with different initial conditions of the system (1) with $R_{0}=0.8163$.


Figure 2. Time series solution with different initial conditions of the system (1) with $R_{0}=8.163>1$. 


\subsection{Sensitivity analysis of $\boldsymbol{R}_{0}$}

In order to discover the key parameters that have a high impact on disease, we present the sensitivity indices of $R_{0}$ to the given set of parameters. We follow the methods in [12] to calculate the sensitivity index, which is defined as the ratio of relative change on $R_{0}$ to the relative change on a parameter $p$ as

$$
\Gamma_{p}^{R_{0}}=\frac{\partial \Gamma}{\partial p} \times \frac{p}{\Gamma}
$$

We note that the maximum value of $\Gamma_{p}^{R_{0}}$ is 1 , which implies that an increase (decrease) of $p$ by $a \%$ increase (decrease) $R_{0}$ by $a \%$. The sensitivity indices for the set of parameter values are presented in Table 2 .

Table 2. The sensitivity indices of $R_{0}$

\begin{tabular}{cc}
\hline Parameter & Indeks Sensitivity \\
\hline $\boldsymbol{\beta}$ & $+\mathbf{1}, \mathbf{0 0 0 0}$ \\
$\Lambda_{u}$ & $+\mathbf{1}, \mathbf{0 0 0 0}$ \\
$\gamma$ & $+\mathbf{0 , 9 0 5 7}$ \\
$\mu_{u}$ & $-\mathbf{0 , 0 9 4 3}$ \\
$\mu_{B}$ & $-\mathbf{0 , 9 0 5 7}$ \\
\end{tabular}

Table 2 shows that the most sensitive parameters are the infection rate and the rate of bacterial proliferation. Therefore, sensitivity analysis can predict the appropriate intervention strategy to prevent and control the disease's spread described by the model. In Figure 3, we show the effect of changing parameter $\beta$ on the number of infected macrophages. From Table 2, we know that the sensitivity index of $\beta, \Gamma_{p}^{R_{0}}=1$. We find that, if $\beta$ increase by $10 \%$ from $6 \times 10^{-6}$ to $6.6 \times 10^{-6}$, then $R_{0}$ increase by $10 \%$ from $R_{0}=2.44898$ to $R_{0}=2.693878$. If $\beta$ decrease by $5 \%$ from $6 \times 10^{-6}$ to $5.7 \times 10^{-6}$, then $R_{0}$ decrease by $5 \%$ from $R_{0}=2.44898$ to $R_{0}=2.326531$.

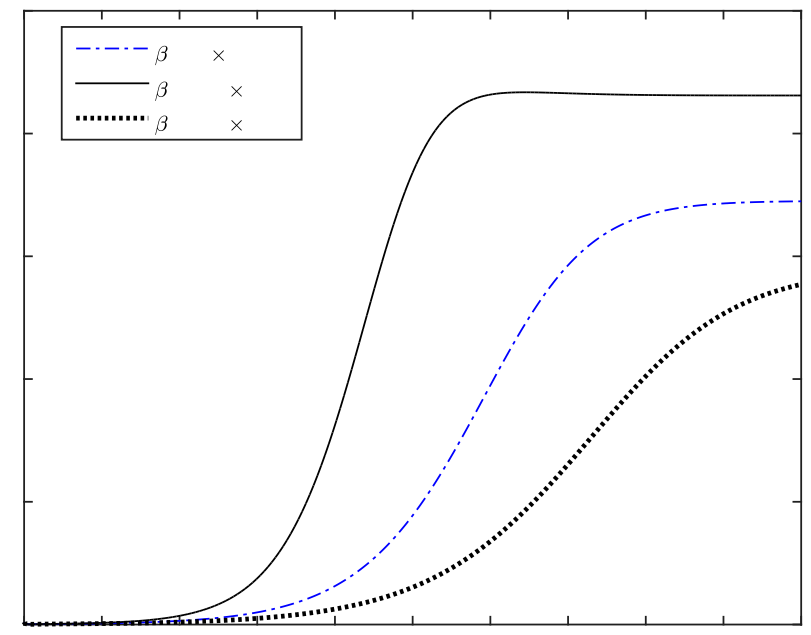

Figure 3. The changing effects of the infection rate $\beta$ on the number of infected macrophages.

Considering the initial conditions $M_{u}(0)=750, M_{i}(0)=10, B(0)=2$, and $T(0)=5$, the results of the simulation are given in Figure 3.

\section{Conclusion}

In this study, we present a theoretical and quantitative analysis of the within-host TB model, taking into account the 
immune response and vaccination. From the mathematical model of the interaction of the immune response to Mycobacterium tuberculosis, two equilibrium points are obtained, namely the disease-free equilibrium point and the infected equilibrium point. The global asymptotically stable disease-free equilibrium point if $R_{0} \leq 1$ and the disease equilibrium point is stable if $R_{0}>1$. From the sensitivity analysis, it is known that the most influential parameters in the spread of tuberculosis are the infection rate $(\beta)$ and bacterial proliferation $(r)$. Medically, TB treatment can be done by paying attention to the two most sensitive parameters. We will carry out a more in-depth study in future research.

\section{Acknowledgments}

Ahmad Dahlan University supports this paper.

\section{References}

[1] WHO. (2019). Global Tuberculosis Control. http://www.who.int/tb/publications/global_report/2019/pdf/fullreport. pdf.

[2] CDC. (2011). The Difference Between Latent TB Infection and Active TB Disease.

[3] CDC. (2016). Transmission and Pathogenesis of Tuberculosis.

[4] Marino, S., Kirschner, D. E. (2004). The human immune response to the Mycobacterium tuberculosis in lung and lymph node. Journal of Theor Biol., vol. 227, pp. 463-486.

[5] Mondragon, E. I., Esteva, L. Galan, L. C. (2011). A Mathematical Model for Cellular Immunology of Tuberculosis, Mathematical Biosciences and Engineering, vol. 8, no. 4, pp. 973-986.

[6] Mondragon, E. I., Esteva, L., Burbano-Rosero, E. M. (2018). Mathematical model for growth of Mycobacterium tuberculosis in granuloma. Math Biosci Eng., vol. 15, no. 2, pp. 407-428.

[7] Shi, R., Li, Y., Tang, S. (2014). A mathematical model with optimal controls for cellular immunology of tuberculosis. Taiwanese Journal of Mathematics, vol. 18, no. 2, pp. 575-597.

[8] Magombedze, G., Garira, W., Mwenje, E. (2006). Modelling the human immune response mechanisms to Mycobacterium tuberculosis infection in lungs. Math Biosci Eng., vol. 3, pp. 661-682.

[9] Zhang, W., Frascoli, F., Heffernan, J. M. (2020). Analysis of solutions and disease progressions for a within-host tuberculosis model. Mathematics in Applied Sciences and Engineering, vol. 1, no. 1, pp. 39-49.

[10] Adi, Y. A., Thobirin, A. (2020). Backward bifurcation in a within-host tuberculosis model. Advances in Mathematics Scientific Journal, vol. 9, no. 9, pp. 7269-7282.

[11] Khalil, H. K. (2002). Nonlinear System. Prentice-Hall, New Jersey.

[12] Chitnis, N., Hyman, J. M., Cushing, J. M. (2008). Determining important parameters in the spread of malaria through the sensitivity analysis of a mathematical model. Bulletin of Mathematical Biology, vol. 70, no. 5, pp. 1272-1296. 